\title{
A 3D Simulation of a Moving Solid in Viscous Free-Surface Flows by Coupling SPH and DEM
}

\author{
Liu-Chao Qiu, ${ }^{1}$ Yi Liu, ${ }^{2}$ and Yu Han ${ }^{1}$ \\ ${ }^{1}$ College of Water Resources \& Civil Engineering, China Agricultural University, Beijing 100083, China \\ ${ }^{2}$ State Key Laboratory of Simulation and Regulation of Water Cycle in River Basin, China Institute of Water Resources and \\ Hydropower Research, Beijing 100038, China \\ Correspondence should be addressed to Liu-Chao Qiu; qiuliuchao@cau.edu.cn and Yu Han; yh916@uowmail.edu.au
}

Received 30 September 2016; Revised 14 November 2016; Accepted 19 December 2016; Published 10 January 2017

Academic Editor: Payman Jalali

Copyright (C) 2017 Liu-Chao Qiu et al. This is an open access article distributed under the Creative Commons Attribution License, which permits unrestricted use, distribution, and reproduction in any medium, provided the original work is properly cited.

\begin{abstract}
This work presents a three-dimensional two-way coupled method to simulate moving solids in viscous free-surface flows. The fluid flows are solved by weakly compressible smoothed particle hydrodynamics (SPH) and the displacement and rotation of the solids are calculated using the multisphere discrete element method (DEM) allowing for the contact mechanics theories to be used in arbitrarily shaped solids. The fluid and the solid phases are coupled through Newton's third law of motion. The proposed method does not require a computational mesh, nor does it rely on empirical models to couple the fluid and solid phases. To verify the numerical model, the floating and sinking processes of a rectangular block in a water tank are simulated, and the numerical results are compared with experimental results reported in published literatures. The results indicate that the method presented in this paper is accurate and is capable of modelling fluid-solid interactions with a free-surface.
\end{abstract}

\section{Introduction}

Fluid-solid interaction in free-surface flows has gained a lot of interests in recent years due to its involvement in a wide variety of industrial processes and engineering disciplines such as hydraulic, ocean, and environmental engineering. Accurate numerical simulations are helpful in obtaining detailed information of the interaction process and in improving the engineering design while avoiding tedious and timeconsuming physical experiments. There are generally two types of coupling model [1] applied to calculate the interaction between fluid and solids: one-way coupling and twoway coupling. Substantial efforts have been made in the past to develop numerical methods for two-way coupling of fluid and solid. For example, Singh et al. [2] used the distributed Lagrange multiplier method for particulate flows with collisions. Takizawa et al. [3] modelled fluid-solid interactions with free-surface flows using CIP method. Latham et al. [4] simulated discrete solids in motion within fluids by coupling of DEM and CFD based on the arbitrary LagrangianEulerian (ALE) frame. Wu et al. [5] presented a two-way coupled Moving Solid Algorithm (MSA) to calculate the motion of the solids in free-surface flows. In contrast to the above-mentioned mesh-based techniques, meshless methods do not require an interface capturing scheme or the moving mesh technology, which is a clear advantage when the problem involves breaking waves and moving boundary. Of the meshless techniques now available, smoothed particle hydrodynamics (SPH) is proving to be popular and robust. It was originally developed for astrophysical simulations $[6,7]$ and has been extended to simulate free-surface flows by Monaghan [8]. Instead of using a mesh, the SPH method uses a set of interpolation nodes placed arbitrarily within the fluid. This gives several advantages in comparison to mesh-based methods when simulating nonlinear flow phenomena. More complete reviews on SPH can be found at $[9,10]$. Attempts have been made in most recently to develop SPH based method for two-way coupling of solid and fluid in a freesurface flow. Hashemi et al. [11] modelled free floating bodies using a ghost particle method which is usually used for fairly regular boundaries. Liu et al. [12] simulated fluid-structure coupling problems, especially for moving structures, using 
incompressible smoothed particle hydrodynamics (ISPH) model. Ren et al. [13] developed a two-dimensional SPHDEM model to simulate the wave-structure interaction by describing the movement of the solids based on multisphere DEM introduced by Favier et al. [14]. Canelas et al. [15] coupled SPH and DEM to describe the motion of arbitrarily shaped solids in viscous fluids based on the concept of DCDEM introduced by Cummins and Cleary [16]. Yang et al. [17] developed an improved SPH-EBG coupling method for modelling the interaction of fluid with free-surface and flexible structure with free and fixed ends.

This work presents a 3D coupled DEM-WCSPH method to simulate moving solids in viscous free-surface flows. The fluid flow was solved by a weakly compressible smoothed particle hydrodynamics (WCSPH) which has an advantage of avoiding solution of the pressure Poisson equation. The motion of the solids was determined by the discrete element method and does not need to be prescribed beforehand. The DEM is also a Lagrangian method which was developed by Cundall and Strack [18] to describe granular material and nowadays is widely used in particulate flows. The DEM model in our study has been implemented using a multisphere approach introduced by Favier et al. [14] to enable solids with different complex shapes to be modelled. The accuracy of the simulation results was verified by comparison with published laboratory experiments of falling and floating solids in water.

\section{Methodology}

2.1. SPH for Fluid Phase. For the fluid phase, the following continuity and momentum equations are employed and given by

$$
\begin{aligned}
& \frac{d \rho}{d t}=-\rho \nabla \mathbf{v}, \\
& \frac{d \mathbf{v}}{d t}=-\frac{1}{\rho} \nabla p+v \nabla^{2} \mathbf{v}+\mathbf{g},
\end{aligned}
$$

where $t$ is the time, $\mathbf{v}$ is the velocity, $p$ is the pressure, $\mathbf{g}$ is gravity acceleration, $v$ is the kinetic viscosity, and $\rho$ is the density.

The key idea of SPH is that the concerning fluid domain is discretized by use of the moving particles which carry all the properties, such as mass, density, velocity, and pressure. And any quantity $A$ of a particle can be approximated by summing over the relevant quantities of its neighboring particles within its support domain $[9,10]$ :

$$
A\left(\mathbf{r}_{a}\right)=\sum_{b} \frac{m_{b}}{\rho_{b}} A\left(\mathbf{r}_{b}\right) W\left(r_{a b}, h\right),
$$

where $m, \rho$, and $\mathbf{r}$ are, respectively, the mass, density, and position of a particle, $h$ is the smoothing length, $r_{a b}=\left|\mathbf{r}_{a}-\mathbf{r}_{b}\right|$ being the distance between particles $a$ and $b$, and $W$ is the smoothing kernel. In general, the higher the order of the kernels, the greater the accuracy of the SPH scheme. The Wendland kernel has some advantages over the Gaussian and the Cubic Spline [19]. So, it is used in this study and written by [20]

$$
W\left(r_{a b}, h\right)=\alpha_{D}\left(1-\frac{q}{2}\right)^{4}(2 q+1), \quad 0 \leq q \leq 2,
$$

where $\alpha_{D}$ is $7 / 4 \pi h^{2}$ in $2 \mathrm{D}$ and $7 / 8 \pi h^{3}$ in $3 \mathrm{D}, q=r_{a b} / h$.

Following Morris et al. [21] and Lo and Shao [22], (1) can be written in SPH discretization as

$$
\begin{aligned}
\frac{d \rho_{a}}{d t}= & \sum_{b} m_{b} \mathbf{v}_{a b} \nabla_{a} W_{a b}, \\
\frac{d \mathbf{v}_{a}}{d t}= & \mathbf{g}_{a}-\sum_{b} m_{b}\left(\frac{p_{a}}{\rho_{a}^{2}}+\frac{p_{b}}{\rho_{b}^{2}}\right) \nabla_{a} W_{a b} \\
& +\sum_{b} m_{b}\left(\frac{4 v \mathbf{r}_{a b} \nabla_{a} W_{a b}}{\left(\rho_{a}+\rho_{b}\right)\left|\mathbf{r}_{a b}\right|^{2}}\right) \mathbf{v}_{a b},
\end{aligned}
$$

where $\mathbf{v}_{a b}=\mathbf{v}_{a}-\mathbf{v}_{b}$, and $\nabla_{a}$ denotes the gradient taken with respect to the coordinates of particle $a$.

As mentioned before, the fluid phase is treated as weakly compressible where the density in the fluid is allowed to vary slightly. The closure problem is solved by using an equation of state to relate changes in pressure to changes in density. Following Monaghan [8] and Batchelor [23], the relationship between pressure and density for a particle $a$ is given by

$$
p_{a}=B\left[\left(\frac{\rho_{a}}{\rho_{0}}\right)^{\gamma}-1\right],
$$

where constant $B=c_{0}^{2} \rho_{0} / \gamma, \rho_{0}$ is the reference density, $c_{0}$ is the sound speed at the reference density, and $\gamma=7$ for a fluid like water. The speed of sound $c_{0}$ is generally chosen as 10 times the maximum velocity in the fluid to ensure the fluctuations in density are less than $1 \%$.

When solving any boundary value problem, the proper implementation of boundary conditions is crucial. Due to its Lagrangian description and to kernel truncation, it is challenging to implement solid boundaries in SPH. In this work, the so-called dynamic boundary conditions [24] are used for both the moving and fixed boundary. It consists of creating boundary particles that satisfy the same equations of continuity (4) and state (6) as the fluid particles, but their positions are not updated using the momentum equation (5) and they remain fixed in position (fixed boundaries) or move according to some externally imposed function (moving objects).

Finally, particle positions updated every time step using the XSPH variant [10]:

$$
\frac{d \mathbf{r}_{a}}{d t}=\mathbf{v}_{a}+\varepsilon \sum_{b} \frac{m_{b}}{\bar{\rho}_{a b}} \mathbf{v}_{a b} \nabla_{a} W_{a b},
$$

where $\bar{\rho}_{a b}=\left(\rho_{a}+\rho_{b}\right) / 2$ and $\varepsilon$ is a constant, whose values range between zero and unity, and $\varepsilon=0.5$ is often used. This method is a correction for the velocity of particle $a$. This correction lets particles to be more organized and, for high fluid velocities, helps to avoid particle penetration. 
2.2. DEM for Solid Phase. DEM coupled with SPH has been used to numerically model the moving solids in viscous freesurface flows. The motion of each solid is tracked based on Newton's laws of motion as follows:

$$
\begin{aligned}
M_{I} \frac{d \mathbf{U}_{I}}{d t} & =\mathbf{F}_{I}^{c}+\mathbf{F}_{I}^{f}+M_{I} \mathbf{g}, \\
I_{I} \frac{d \mathbf{\Omega}_{I}}{d t} & =\mathbf{T}_{I},
\end{aligned}
$$

where solid $I$ possesses mass $M_{I}$, velocity $\mathbf{U}_{I}$, and angular velocity $\boldsymbol{\Omega}_{I} \cdot \mathbf{g}$ is gravity acceleration. Force $\mathbf{F}_{I}^{f}$ is due to fluidsolid interaction and force $\mathbf{F}_{I}^{c}$ represents any solid contact that might occur. Integrating (8) into time advances the linear motion of the solid, whereas (9) accounts for the rotational motion.

In order to calculate the fluid-solid interaction force $\mathbf{F}_{I}^{f}$, the boundary of a moving solid is represented by groups of $\mathrm{SPH}$ particles and these moving boundary particles have an interparticle spacing that is equal to half of the fluid particle spacing to prevent the fluid particles from penetrating the moving solid boundary. The fluid-solid interaction is ensured through the interactive force balance condition satisfying Newton's third law of motion. Following Ren et al. [13], the fluid-solid interaction force $\mathbf{F}_{I}^{f}$ can be determined as

$$
\mathbf{F}_{I}^{f}=\sum_{i} \sum_{a} m_{i} m_{a}\left(\frac{p_{a}}{\rho_{a}^{2}}+\frac{p_{i}}{\rho_{i}^{2}}\right) \nabla_{a} W_{a i},
$$

where the inner summation means the total force on moving boundary particle $i$ belonging to solid $I$ due to the neighborhood fluid particle $a$.

Following Latham et al. [4], a multisphere approach originally proposed by Favier et al. [14] was used for modelling the complex-shaped multibody dynamics in which the surface of each solid is represented by a cluster of small spheres whose diameter is equivalent to the spacing of the moving boundary particles. The solids are allowed to interact via contact forces when the surface spheres of different solids overlap. No relative movement between spheres of the same body is allowed. Based on the multisphere approach, the resultant contact forces and torque acting upon solid $I$ are evaluated as

$$
\begin{aligned}
& \mathbf{F}_{I}^{c}=\sum_{i} \sum_{j}\left(\mathbf{f}_{i j, n}+\mathbf{f}_{i j, t}\right), \\
& T_{I}=\sum_{i} \sum_{j}\left(R_{i} \mathbf{n}_{i j} \times \mathbf{f}_{i j, t}\right),
\end{aligned}
$$

where $R_{i}$ is the radius of surface sphere $i$ belonging to $I$ and $\mathbf{n}_{i j}$ represents the normal unit vector pointing from the center of sphere $i$ belonging to $I$ to the center of sphere $j$ belonging to $J$. $\mathbf{f}_{i j, n}$ and $\mathbf{f}_{i j, t}$ are the normal and tangential forces, respectively, on surface sphere $i$ of solid $I$ due to surface sphere $j$ of solid $J$. Based on the model by Cundall and Strack [18], the normal contact forces $\mathbf{f}_{i j, n}$ are calculated by

$$
\mathbf{f}_{i j, n}=-k_{n} \delta_{n} \mathbf{n}_{i j}-\eta_{n} \mathbf{v}_{i j, n},
$$

where $k_{n}$ is the normal spring stiffness, the overlap $\delta_{n}=$ $\left(R_{i}+R_{j}\right)-\left|\mathbf{x}_{i}-\mathbf{x}_{j}\right|$, where $R_{i}$ and $R_{j}$ are the surface sphere radii, and $\mathbf{x}_{i}$ and $\mathbf{x}_{j}$ are the surface sphere centers. $\mathbf{v}_{i j, n}$ is the normal relative velocity, and $\eta_{n}$ is the normal damping coefficient given by

$$
\eta_{n}=-\frac{2 \log e_{i j} \sqrt{m^{*} k_{n}}}{\left(\log e_{i j}\right)^{2}+\pi^{2}}
$$

where $e_{i j}$ is the restitution coefficient. The effective mass $m^{*}=$ $\left(m_{i}+m_{j}\right) / m_{i} m_{j}$, where $m_{i}$ and $m_{j}$ are the mass of surface sphere.

The tangential component of the contact force $\mathbf{f}_{i j, t}$ is calculated by a Coulomb friction law using a coefficient of friction $\mu_{i j}$. It can be expressed as

$$
\mathbf{f}_{i j, t}= \begin{cases}-k_{t} \delta_{t} \mathbf{t}_{i j}-\eta_{t} \mathbf{v}_{i j, t} & \left|\mathbf{f}_{i j, t}\right|<\mu_{i j}\left|\mathbf{f}_{i j, n}\right| \\ -\mu_{i j}\left|\mathbf{f}_{i j, n}\right| \mathbf{t}_{i j} & \left|\mathbf{f}_{i j, t}\right|>\mu_{i j}\left|\mathbf{f}_{i j, n}\right|,\end{cases}
$$

where $k_{t}, \delta_{t}$, and $\eta_{t}$ are the tangential spring stiffness, tangential overlap, and tangential damping coefficient, respectively. The tangential relative velocity $\mathbf{v}_{i j, t}=\mathbf{v}_{i j}-\mathbf{v}_{i j, n}$ and the tangential unit vector $\mathbf{t}_{i j}=\mathbf{v}_{i j, t} /\left|\mathbf{v}_{i j, t}\right|$. The tangential damping coefficient given by

$$
\eta_{t}=-\frac{2 \log e_{i j} \sqrt{m^{\prime} k_{n}}}{\left(\log e_{i j}\right)^{2}+\pi^{2}}
$$

where $k_{t}=(2 / 7) k_{n}$ and $m^{\prime}=(2 / 7) m^{*}$. The value of $2 / 7$ causes the normal and tangential springs to react on similar time scales [24].

\section{Numerical Examples and Discussion}

In this section, numerical examples are presented to validate both solid-solid and fluid-solid interactions. For the cases involving water, the initial velocity of the fluid particles is considered zero. The fluid particles are assigned an initial density based on hydrostatic pressure. Therefore, the density of particle $a$ (located at depth $z$ ) is calculated taking in account of the water column height as

$$
\rho(x, y, z)=\rho_{0}\left(1+\frac{\rho_{0} g(H(x, y)-z)}{B}\right)^{\gamma},
$$

where $H(x, y)$ is the initial water depth at position $(x, y)$ and $z$ is the vertical distance from the bottom. The pressure is calculated using equation of state (6) following the density value. In all the simulations presented herein, the modelled fluid is water with reference density $\rho_{0}=1000 \mathrm{~kg} / \mathrm{m}^{3}$ and kinetic viscosity $v=1 \times 10^{-6} \mathrm{~m}^{2} / \mathrm{s}$. For all simulations, the smoothing length is defined as $h=1.2\left(\Delta x^{2}+\Delta y^{2}+\Delta z^{2}\right)^{1 / 2}$, where $\Delta x=\Delta y=\Delta z=\Delta d$ represent the initial particle spacing.

3.1. Solid Dropping onto a Flat Floor. In this simulation, a sphere is dropped under the influence of gravity onto a flat 


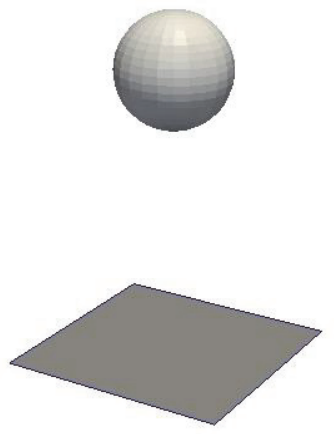

(a)

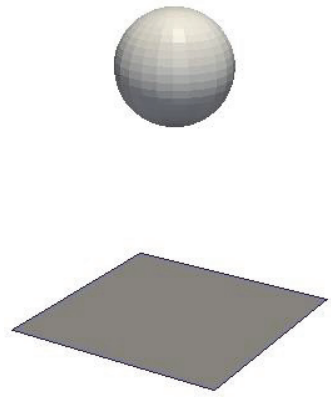

(b)

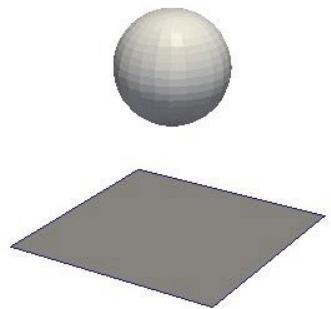

(c)

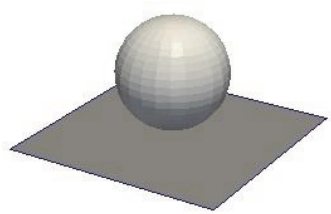

(d)

FIGURE 1: Snapshots of the falling ball at different times: (a) $t=0 \mathrm{~s}$, (b) $t=0.1 \mathrm{~s}$, (c) $t=0.2 \mathrm{~s}$, and (d) $t=0.28 \mathrm{~s}$.

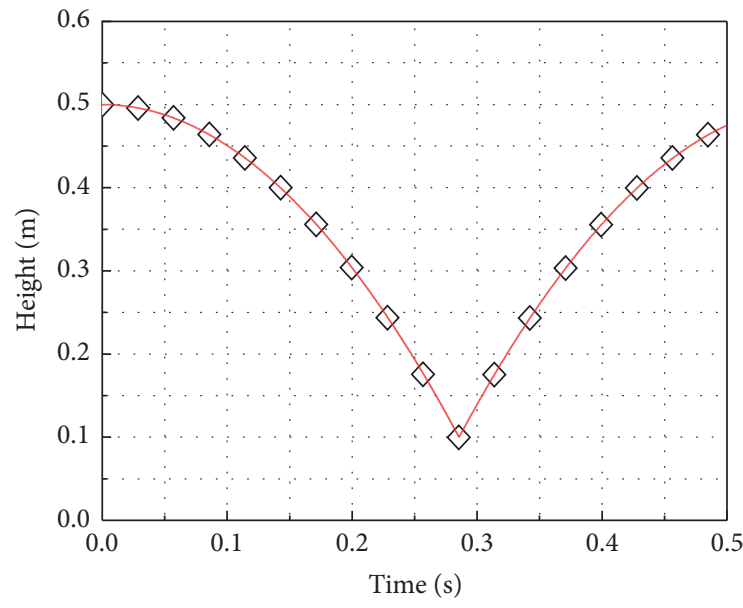

$\diamond$ Numerical - Analytical

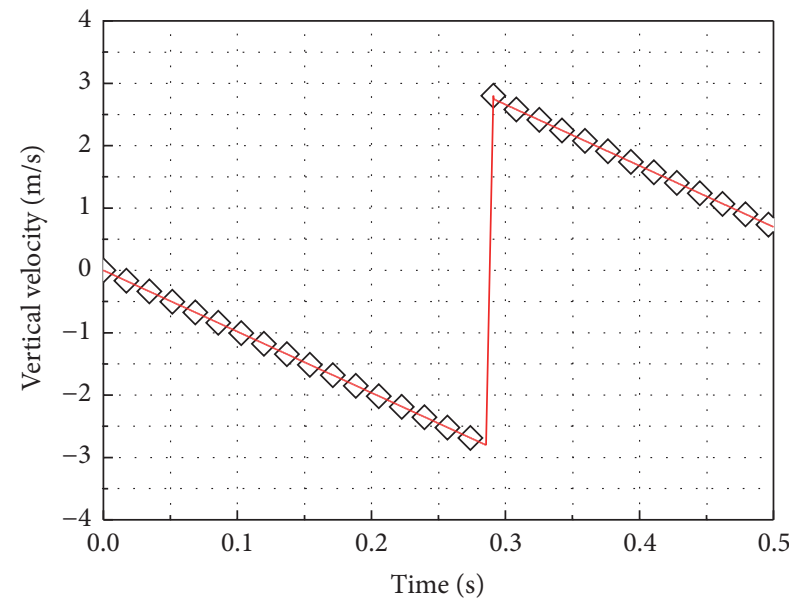

$\diamond$ Numerical

Analytical

FIGURE 2: Comparison between analytic solution and numerical results.

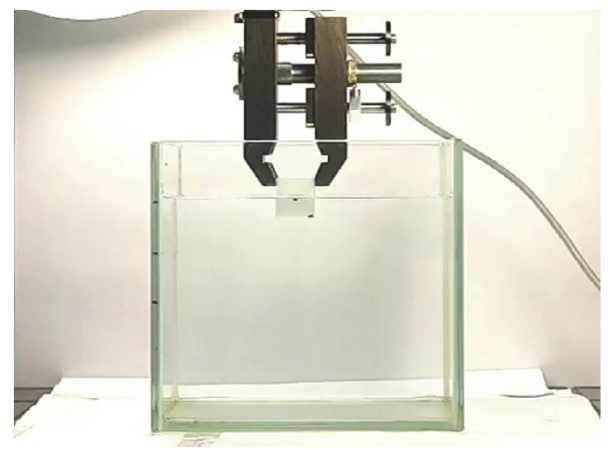

FIGURE 3: Experimental setup of the falling cube (after Wu et al. [5]).

floor. The purpose of this simulation is to verify that the momentum exchange between a solid and a flat boundary agrees with theory based on the principle of impulse and also to see if the motion of the solid subject to a gravitational force is accurate. The problem contains one sphere whose bottom is

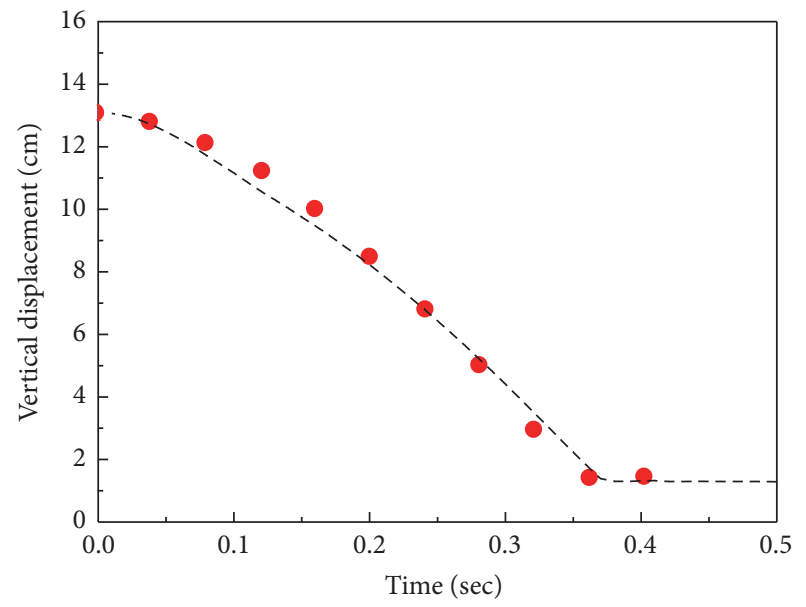

- Experiment -.- Simulation

FIGURE 4: Comparison of measured and predicted vertical displacements. 

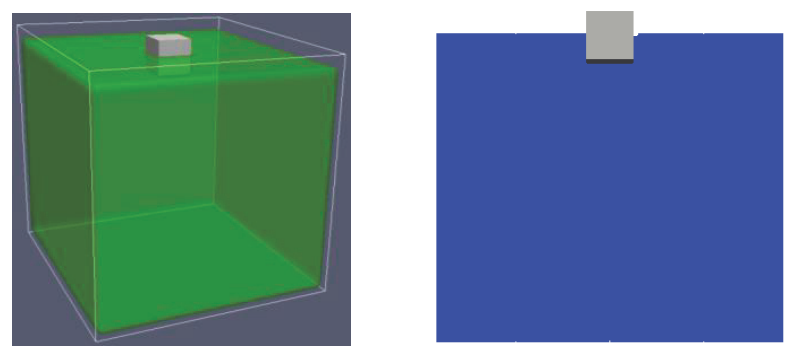

(a) $t=0.0 \mathrm{sec}$
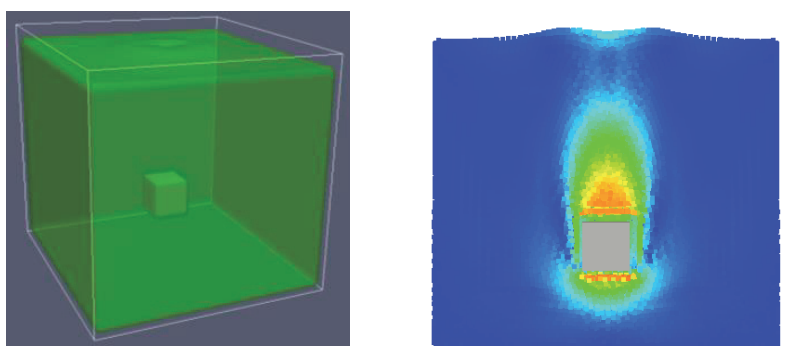

(c) $t=0.3 \mathrm{sec}$
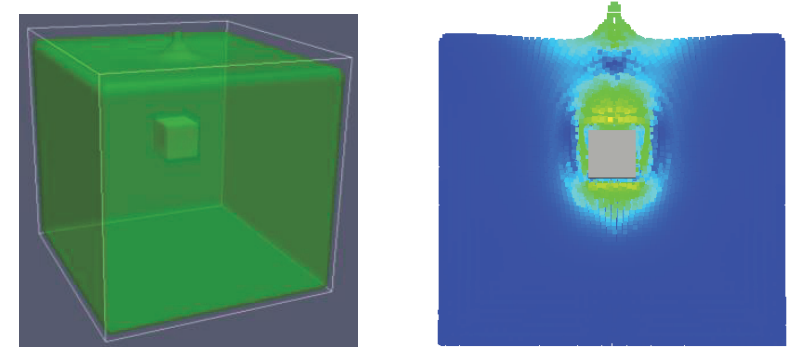

(b) $t=0.2 \mathrm{sec}$
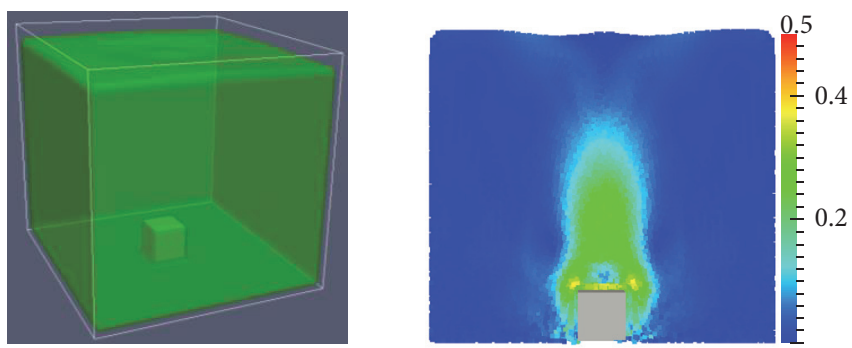

(d) $t=0.4 \mathrm{sec}$

Figure 5: The falling cube in the water tank with plotting the velocity magnitude.

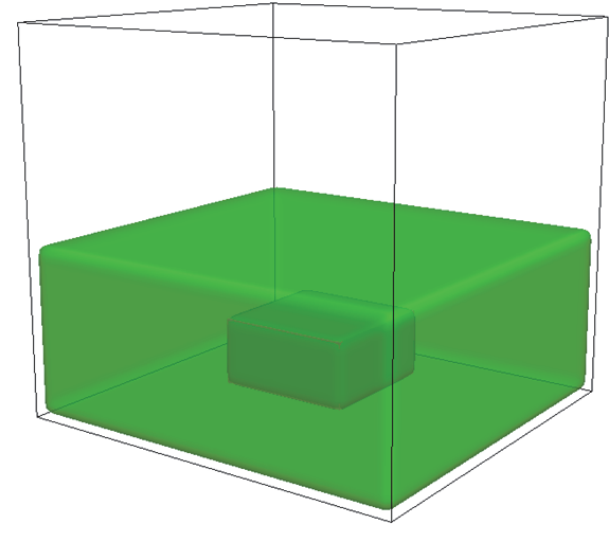

FIGURE 6: Initial configuration of the floating solid problem.

$0.5 \mathrm{~m}$ above a flat floor. The solid sphere has a density of $2.6 \times$ $10^{3} \mathrm{~kg} / \mathrm{m}^{3}$, a diameter of $0.1 \mathrm{~m}$, and a normal spring stiffness of $1 \times 10^{5} \mathrm{~N} / \mathrm{m}$. The collision is assumed to be frictionless and purely elastic. So, coefficient of friction $\mu=0$ and the restitution coefficient $e=1$. A time step of $1.0 \times 10^{-4} \mathrm{~s}$ and an initial fluid particle spacing $\Delta d=0.01 \mathrm{~m}$ are used in the simulation. The sphere is dropped and falls under the force of gravity until it contacts the level surface. The translational motion of the sphere can be described in three stages: free fall, contact, and rebound. Figure 1 shows snapshots of the falling sphere at different time. Figure 2 compares the vertical velocity and the height of the solid as a function of time. The results agree very well with the analytical solution given in the work of Chen et al. [25].

3.2. Falling Solid in Water. The aim of this simulation is to evaluate the fluid-solid coupling of a solid in a free-surface

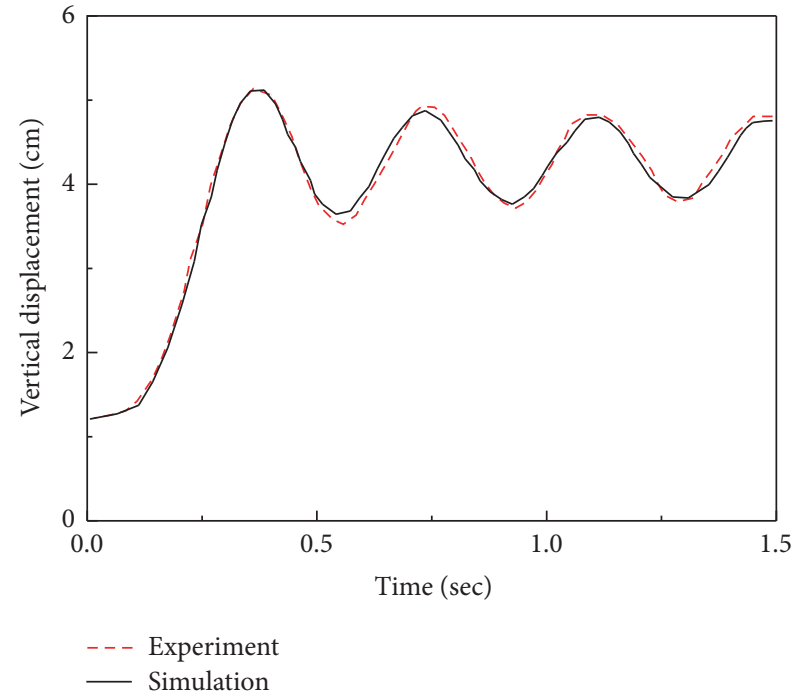

FIGURE 7: Comparison of measured and predicted vertical displacement of the floating solid.

flow. The simulation involves a cube falling into calm water in a tank. The trajectory of the cube is compared to laboratory experiments proposed by $\mathrm{Wu}$ et al. [5]. In their experiments, a cube was released by a clamp at the water surface and the trajectory of the cube was recorded by a high speed camera. In order to reduce the water splash caused by the cube falling into water, the bottom of the cube was immersed in the water before the cube was released. The clamp was controlled by an electromagnetic device so the cube could be released instantly (see Figure 3).

The problem domain contains a water tank of $150 \mathrm{~mm} \times$ $140 \mathrm{~mm} \times 140 \mathrm{~mm}$, and the water depth in the tank was 

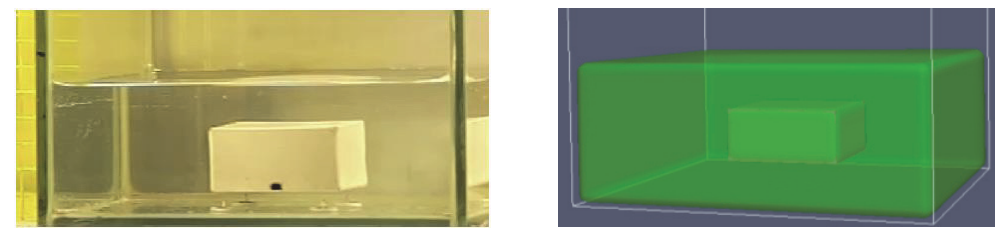

(a) $t=0.11 \mathrm{sec}$
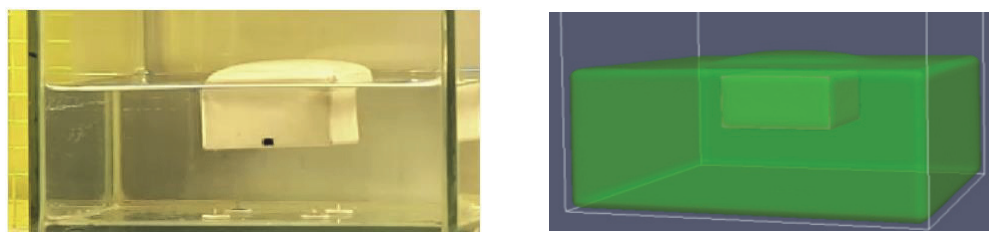

(b) $t=0.25 \mathrm{sec}$
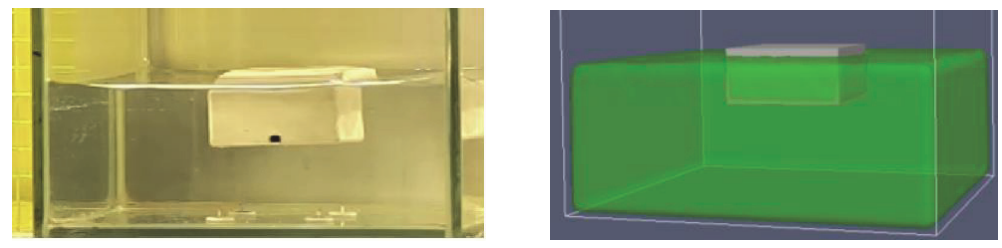

(c) $t=0.61 \mathrm{sec}$

FIGURE 8: Snapshots of numerical (left) and experimental (right) results of the floating block.

$131 \mathrm{~mm}$. The cube with a side length of $20 \mathrm{~mm}$ was assumed be a rigid as in the experiment. The density of the cube was $2120 \mathrm{~kg} / \mathrm{m}^{3}$, the normal spring stiffness was $1 \times 10^{5} \mathrm{~N} / \mathrm{m}$, the coefficient of friction $\mu=0$, and the restitution coefficient $e=0.5$. A time step of $1.0 \times 10^{-4} \mathrm{~s}$ and an initial fluid particle spacing $\Delta d=2 \mathrm{~mm}$ are used in the simulation. Figure 4 compares the vertical displacements of the cube to the experimental results in $\mathrm{Wu}$ et al. [5]. The simulation results are in good agreement compared with the measured trajectory in the vertical direction. Figure 5 presents some snapshots at different instants of falling cube evolution with the velocity magnitude $\left(v=\sqrt{v_{x}^{2}+v_{y}^{2}+v_{z}^{2}}\right)$ on the central cutting section. The color bar is common to all snapshots and velocities are in meters per second.

3.3. Floating Solid in Water. In this simulation, a wooden block rising from the bottom of a water tank was considered. Figure 6 shows the initial configuration of the problem. The numerical results are also compared with the experimental data provided by $\mathrm{Wu}$ et al. [5]. The size of the water tank was $150 \mathrm{~mm} \times 140 \mathrm{~mm} \times 140 \mathrm{~mm}$, and the water depth in the tank was $52 \mathrm{~mm}$. A rectangular wooden block (width $=48 \mathrm{~mm}$, length $=49 \mathrm{~mm}$, height $=24 \mathrm{~mm}$, and density $800.52 \mathrm{~kg} / \mathrm{m}^{3}$ ) was released from the bottom of the water tank. In our simulation, the normal spring stiffness was $1 \times 10^{5} \mathrm{~N} / \mathrm{m}$, the coefficient of friction $\mu=0$, and the restitution coefficient $e=0.5$. A time step of $1.0 \times 10^{-4} \mathrm{~s}$ and an initial fluid particle spacing $\Delta d=2 \mathrm{~mm}$ are used in the simulation. Figure 7 shows the comparison of measured and predicted vertical displacements of the block. It shows a good agreement between the simulated results and experimental data by $\mathrm{Wu}$ et al. [5]. Figure 8 shows the snapshots of the configuration of the floating solid at different time and the calculated freesurface profiles are very similar to that of the laboratory results.

\section{Conclusions}

A three-dimensional numerical model has been developed to simulate moving solids interacting with a free-surface flow by coupling the smoothed particle hydrodynamics and the discrete element method. The motions of the solids do not need to be prescribed beforehand and the mesh-free method is used for the computational fluid dynamics. The DEM model has been implemented using a multisphere approach to enable solids with arbitrarily complex shapes to be modelled. The comparison of results from the coupled approach with known analytical solutions and experimental data indicates that the proposed methods could be an efficient way of simulating complex moving solids in viscous freesurface flows. Our numerical examples focus mainly on fluidsolid interaction; however, multibody systems with solidsolid contacts are also within the capabilities of the model. Further investigations might be needed to consider the solid deformable and turbulent effects.

\section{Competing Interests}

The authors declare that there are no competing interests regarding the publication of this paper.

\section{Acknowledgments}

The authors are grateful for funding from the National Natural Science Foundation of China (Grant nos. 11172321 
and 51509248), the Open Fund of the State Key Laboratory of Hydroscience and Engineering of China, Tsinghua University (Grant no. sklhse-2015-C-03), the Scientific Research and Experiment of Regulation Engineering for the Songhua River Mainstream in Heilongjiang Province, China (Grant no. SGZL/KY-12), and the Open Fund of the State Key Laboratory of Simulation and Regulation of Water Cycle in River Basin, China Institute of Water Resources and Hydropower Research (Grant no. IWHR-SKL-201505).

\section{References}

[1] J. F. O'Brien, V. B. Zordan, and J. K. Hodgins, "Combining active and passive simulations for secondary motion," IEEE Computer Graphics and Applications, vol. 20, no. 4, pp. 86-96, 2000.

[2] P. Singh, T. I. Hesla, and D. D. Joseph, "Distributed Lagrange multiplier method for particulate flows with collisions," International Journal of Multiphase Flow, vol. 29, no. 3, pp. 495-509, 2003.

[3] K. Takizawa, T. Yabe, Y. Tsugawa, T. E. Tezduyar, and H. Mizoe, "Computation of free-surface flows and fluid-object interactions with the CIP method based on adaptive meshless soroban grids," Computational Mechanics, vol. 40, no. 1, pp. 167-183, 2007.

[4] J.-P. Latham, A. Munjiza, J. Mindel et al., "Modelling of massive particulates for breakwater engineering using coupled FEMDEM and CFD," Particuology, vol. 6, no. 6, pp. 572-583, 2008.

[5] T.-R. Wu, C.-R. Chu, C.-J. Huang, C.-Y. Wang, S.-Y. Chien, and M.-Z. Chen, "A two-way coupled simulation of moving solids in free-surface flows," Computers \& Fluids, vol. 100, pp. 347-355, 2014.

[6] L. B. Lucy, "A numerical approach to the testing of the fission hypothesis," The Astronomical Journal, vol. 82, pp. 1013-1024, 1977.

[7] R. A. Gingold and J. J. Monaghan, "Smoothed particle hydrodynamics: theory and application to non-spherical stars," Monthly Notices of the Royal Astronomical Society, vol. 181, no. 3, pp. 375389, 1977.

[8] J. J. Monaghan, "Simulating free surface flows with SPH," Journal of Computational Physics, vol. 110, no. 2, pp. 399-406, 1994.

[9] G. R. Liu and M. B. Liu, Smoothed Particle Hydrodynamics: A Mesh Free Particle Method, World Scientific, 2003.

[10] J. J. Monaghan, “Smoothed particle hydrodynamics," Reports on Progress in Physics, vol. 68, no. 8, pp. 1703-1759, 2005.

[11] M. R. Hashemi, R. Fatehi, and M. T. Manzari, "A modified SPH method for simulating motion of rigid bodies in Newtonian fluid flows," International Journal of Non-Linear Mechanics, vol. 47, no. 6, pp. 626-638, 2012.

[12] X. Liu, P. Lin, and S. Shao, "An ISPH simulation of coupled structure interaction with free surface flows," Journal of Fluids and Structures, vol. 48, pp. 46-61, 2014.

[13] B. Ren, Z. Jin, R. Gao, Y.-X. Wang, and Z.-L. Xu, "SPH-DEM modeling of the hydraulic stability of $2 \mathrm{D}$ blocks on a slope," Journal of Waterway, Port, Coastal and Ocean Engineering, vol. 140, no. 6, Article ID 04014022, 2014.

[14] J. F. Favier, M. H. Abbaspour-Fard, M. Kremmer, and A. O. Raji, "Shape representation of axi-symmetrical, non-spherical particles in discrete element simulation using multi-element model particles," Engineering Computations (Swansea, Wales), vol. 16, no. 4, pp. 467-480, 1999.
[15] R. B. Canelas, A. J. Crespo, J. M. Domínguez, R. M. Ferreira, and M. Gómez-Gesteira, "SPH-DCDEM model for arbitrary geometries in free surface solid-fluid flows," Computer Physics Communications, vol. 202, pp. 131-140, 2016.

[16] S. J. Cummins and P. W. Cleary, "Using distributed contacts in DEM," Applied Mathematical Modelling, vol. 35, no. 4, pp. 19041914, 2011.

[17] X. Yang, M. Liu, S. Peng, and C. Huang, "Numerical modeling of dam-break flow impacting on flexible structures using an improved SPH-EBG method," Coastal Engineering, vol. 108, pp. 56-64, 2016.

[18] P. A. Cundall and O. D. L. Strack, "A discrete numerical model for granular assemblies," Geotechnique, vol. 29, no. 1, pp. 47-65, 1979.

[19] F. Macia, A. Colagrossi, M. Antuono, and A. Souto-Iglesias, "Benefits of using a wendland kernel for free-surface flows," in Proceedings of the 6th International ERCOFTAC SPHERIC Workshop on SPH Applications, pp. 30-37, Hamburg, Germany, 2011.

[20] H. Wendland, "Piecewise polynomial, positive definite and compactly supported radial functions of minimal degree," Advances in Computational Mathematics, vol. 4, no. 4, pp. 389396, 1995.

[21] J. P. Morris, P. J. Fox, and Y. Zhu, "Modeling low Reynolds number incompressible flows using SPH," Journal of Computational Physics, vol. 136, no. 1, pp. 214-226, 1997.

[22] E. Y. M. Lo and S. Shao, "Simulation of near-shore solitary wave mechanics by an incompressible SPH method," Applied Ocean Research, vol. 24, no. 5, pp. 275-286, 2002.

[23] G. K. Batchelor, Introduction to Fluid Dynamics, Cambridge University Press, 1974.

[24] A. J. C. Crespo, M. Gómez-Gesteira, and R. A. Dalrymple, "Boundary conditions generated by dynamic particles in SPH methods," Computers, Materials, \& Continua, vol. 5, no. 3, pp. 173-184, 2007.

[25] F. Chen, E. C. Drumm, and G. Guiochon, "Prediction/verification of particle motion in one dimension with the discreteelement method," International Journal of Geomechanics, vol. 7, no. 5, pp. 344-352, 2007. 


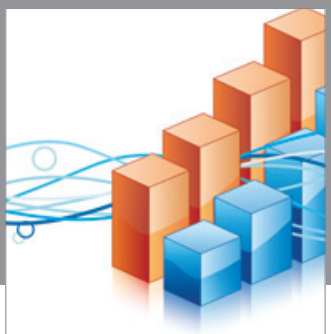

Advances in

Operations Research

vatem alat4

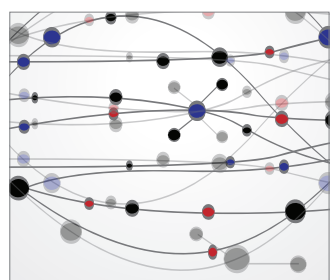

\section{The Scientific} World Journal
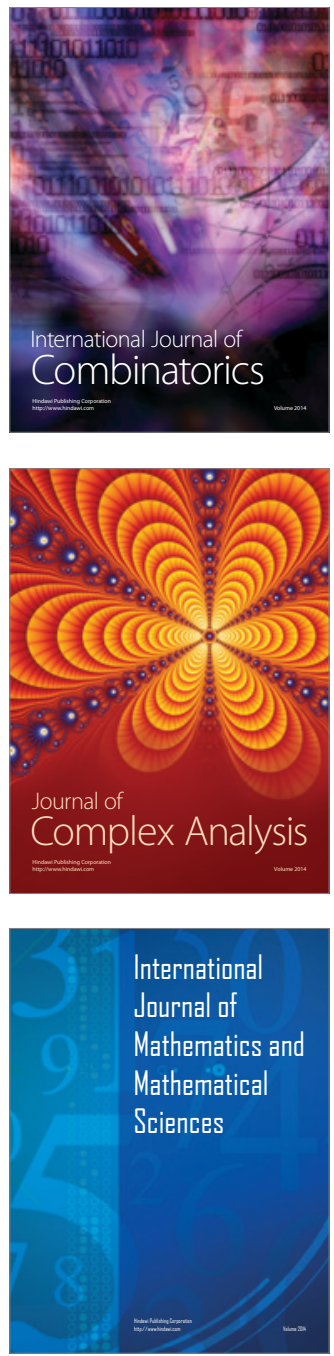
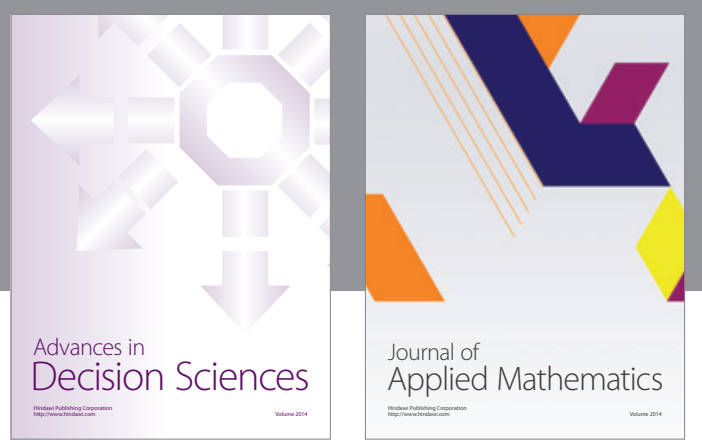

Algebra

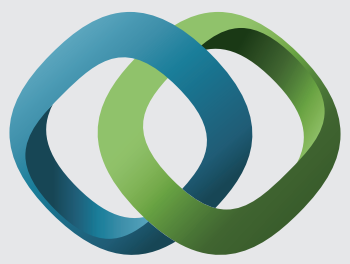

\section{Hindawi}

Submit your manuscripts at

https://www.hindawi.com
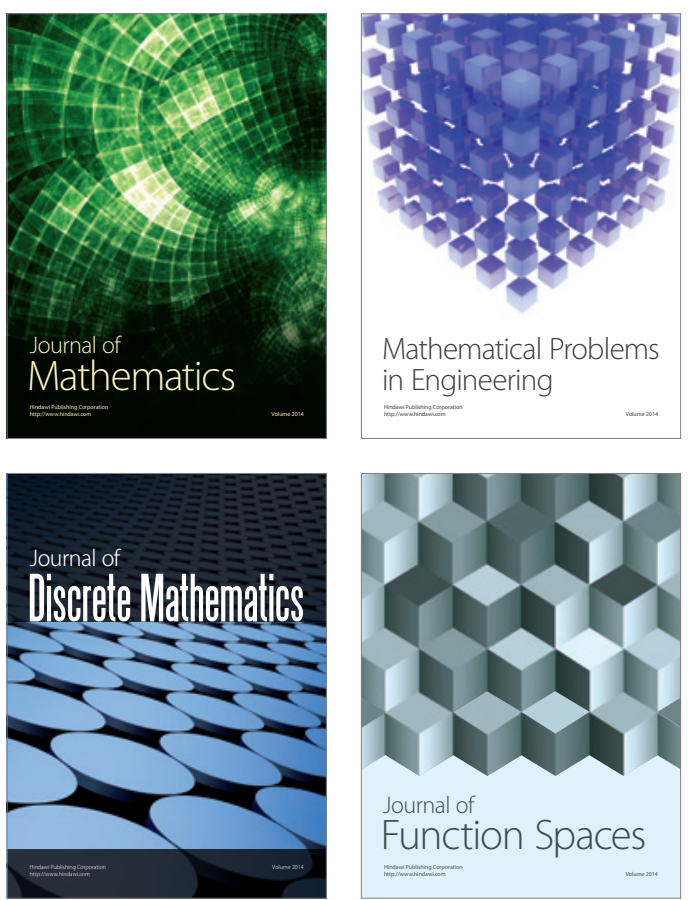

Mathematical Problems in Engineering
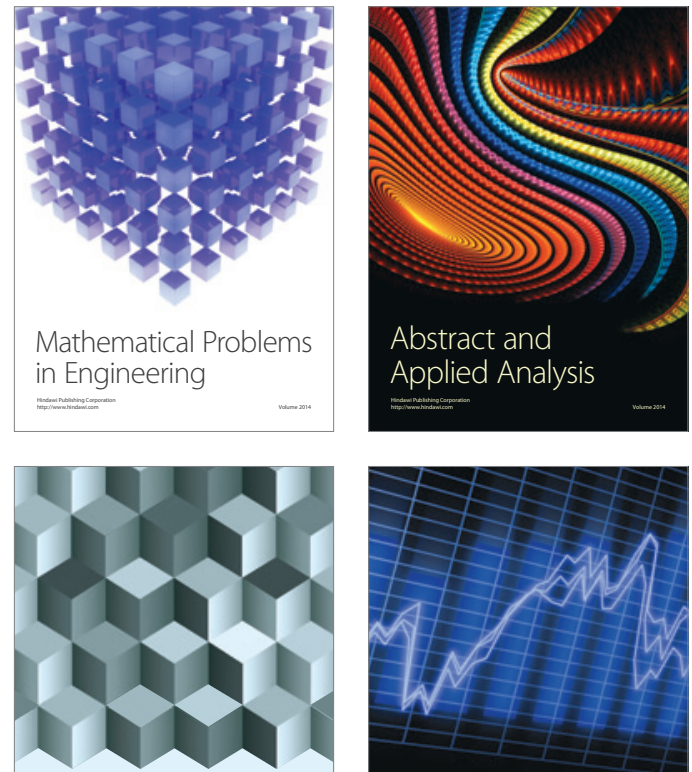

Journal of

Function Spaces

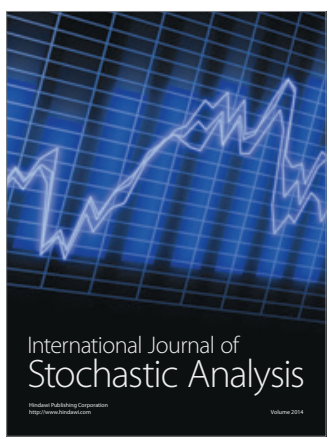

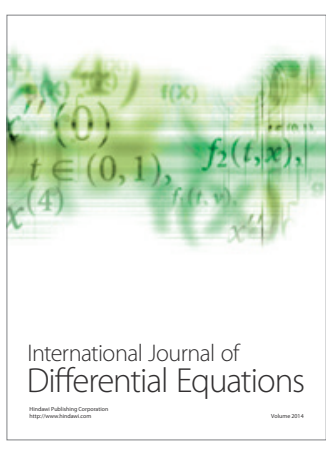
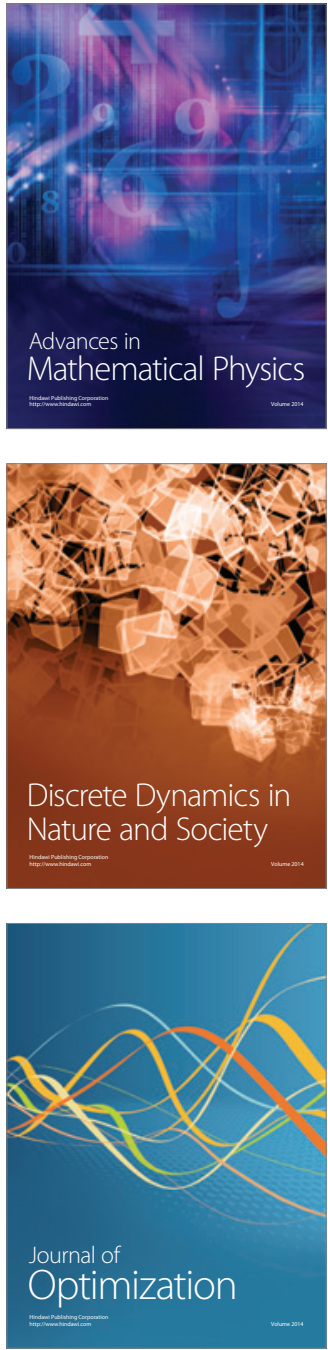